Z Rheumatol 2019.78:568-576

https://doi.org/10.1007/s00393-018-0550-2

○ Der/die Autor(en) 2018

\section{Redaktion}

U. Müller-Ladner, Bad Nauheim

U. Lange, Bad Nauheim

CrossMark

\author{
J. Braun ${ }^{1,2} \cdot$ T. Mosch $^{3} \cdot$ I. Fischer ${ }^{4} \cdot$ U. Kiltz ${ }^{1,2}$ \\ 'Rheumazentrum Ruhrgebiet, Herne, Deutschland \\ ${ }^{2}$ Ruhr Universität Bochum, Bochum, Deutschland \\ ${ }^{3}$ Medical Affairs, AbbVie Deutschland GmbH \& Co KG, Wiesbaden, Deutschland \\ ${ }^{4}$ Biostatistik-Tübingen, Tübingen, Deutschland
}

\title{
Identifikation von Patienten mit axialer Spondyloarthritis in der Primärversorgung (AWARE- Studie)
}

\section{Hintergrund und Fragestellung}

Die axiale Spondyloarthritis (axSpA) gehört zu den häufigsten entzündlich rheumatischen Erkrankungen [30]. Die Klassifikation [24] umfasst die klassische ankylosierende Spondylitis (AS) [8] und die nicht-röntgenologische axSpA (nr-axSpA) [25, 26]. Hauptsymptom ist der entzündliche Rückenschmerz [6]. Diese charakteristische Form des Rückenschmerzes zeichnet sich dadurch aus, dass er bei relativ jungen Patienten auftritt, oft tiefsitzend oder auch als wechselseitiger Gesäßschmerz empfunden wird. Die Schmerzphasen beginnen schleichend, sie treten oft in der zweiten Nachthälfte auf; die Schmerzen lassen sich durch Bewegung, nicht aber durch Ruhe bessern [6]. Wichtig ist, dass sich keines der Charakteristika des entzündlichen Rückenschmerzes als einzelne Variable zur Differenzierung zwischen Patienten mit axSpA und nichtspezifischem Rückenschmerz eignet.

Für die Diagnosestellung einer axSpA wurden Algorithmen entwickelt [5], die kürzlich problematisiert [14] und diskutiert wurden [7]. Auf den wichtigen Unterschied zwischen Klassifikation und Diagnose ist verschiedentlich hingewiesen worden [11]. Für beides, Klassifikation und Diagnose, spielt die Bildgebung mittels konventionellen Röntgens und Magnetresonanztomographie (MRT) der Sakroiliakalgelenke eine wichtige Rolle - für beide liegen konsentier- te Definitionen vor [19, 20]. Während die korrekte Zuordnung und Graduierung von strukturellen Veränderungen im konventionellen Röntgenbild bekanntermaßen problematisch ist [28], mehren sich auch für die MRT Bedenken hinsichtlich der korrekten Zuordnung von Läsionen. So wurde zumindest das differenzialdiagnostische Spektrum des Knochenmarködems erheblich erweitert $[12,29]$.

Nicht nur vor diesem Hintergrund ist die Frühdiagnose der axSpA weiterhin eine erhebliche Herausforderung - auch wenn hierzu bereits Studienergebnisse [ 9 , $10,13,17,21,27]$ und eine vorläufige Empfehlung [22] vorliegen. Ein insgesamt sehr kritisch zu betrachtender Ansatz wurde kürzlich besonders intensiv diskutiert [2, 18]. Hierbei handelte es sich um die Evaluierung eines Kriteriensets für die Entdeckung von axSpAPatienten auf Populationsbasis, bei der für die Diagnosebestätigung die ASASKlassifikationskriterien verwendet wurden, das wird von den meisten Experten zu Recht abgelehnt.

Die erste Anlaufstelle für Patienten mit Rückenschmerzen sind neben Allgemeinmedizinern meist Orthopäden. Im Rahmen einer Pilotstudie (SepyA-Studie) hatte sich die Kombination von bestimmten klinischen Parametern, die typisch für den sog. entzündlichen Rückenschmerz sein können, als nützlich für die Identifikation von axSpA-Patienten in der Primärversorgung erwiesen [9].
Hierbei hatte sich gezeigt, dass das Vorliegen von mindestens 3 von 5 Parametern (s. unten, hier AWARE-Parameter genannt), eine gute Sensitivität und Spezifität für das Vorliegen einer axSpA hat. Ziel der jetzt hier vorgestellten Kohortenstudie war die Überprüfung dieser 5 AWARE-Parameter im Hinblick auf die Identifikation von Patienten mit axSpA innerhalb einer großen Kohorte von jungen Patienten mit chronischen Rückenschmerzen in der orthopädischen Primärversorgung.

\section{Studiendesign und Untersuchungsmethoden}

Die Rekrutierung der Patienten erfolgte durch Orthopäden. Eingeschlossen wurden junge Erwachsene ( $>18$ Jahre) mit chronischen Rückenschmerzen ( $\geq 3$ Monate) und einem Alter bei Beschwerdebeginn <45 Jahre, die - studienunabhängig - nach Einschätzung der Orthopäden zum Rheumatologen überwiesen werden sollten. Im Rahmen der Studiendokumentation wurden neben demografischen und anderen anamnestischen Parametern die sog. AWARE-Parameter („Alter bei Beginn der Rückenschmerzen <35 Jahre“, „Wechselseitiger Gesäßschmerz", „Ansprechen auf nichtsteroidale Antirheumatika (NSAR) innerhalb von $48 \mathrm{~h}$ “, „Regung/Bewegung bringt Besserung“, „Erwachen in der zweiten Nachthälf- 
Tab. 1 Demografische und anamnesti-

sche Angaben Pop-OR-2 $(n=500) /$ Pop-OR-S2 $(n=343)$

\begin{tabular}{|c|c|c|}
\hline Parameter & Mw & Std \\
\hline \multicolumn{3}{|l|}{ Alter (Jahre) } \\
\hline $\begin{array}{l}\text { Pop-OR-2 (Patienten mit } \\
\text { plausibler Diagnose) }\end{array}$ & 38,0 & 11,5 \\
\hline $\begin{array}{l}\text { Pop-OR-S2 (Patienten } \\
\text { mit bestätigtem HLA } \\
\text { B27-Befund) }\end{array}$ & 37,1 & 11,0 \\
\hline \multicolumn{3}{|c|}{ Dauer Rückenschmerz (Monate) } \\
\hline Pop-OR-2 & 94,1 & 103,6 \\
\hline Pop-OR-S2 & 83,7 & 88,6 \\
\hline \multicolumn{3}{|l|}{ Dauer Symptome (Monate) } \\
\hline Pop-OR-2 $(n=497)$ & 83,6 & 95,0 \\
\hline Pop-OR-S2 $(n=340)$ & 81,4 & 95,0 \\
\hline \multicolumn{3}{|l|}{ Männliches Geschlecht in \% } \\
\hline Pop-OR-2 & 46,2 & \\
\hline Pop-OR-S2 & 48,4 & \\
\hline \multicolumn{3}{|c|}{$\begin{array}{l}\text { Population OR-2 alle Patienten, die sowohl } \\
\text { von Orthopäden als auch Rheumatologen } \\
\text { gesehen wurden und die eine plausible } \\
\text { Diagnose hatten; Population OR-S2 alle } \\
\text { Patienten, bei den auch ein HLA B27-Befund } \\
\text { vorlag }\end{array}$} \\
\hline
\end{tabular}

te aufgrund der Rückenschmerzen“) abgefragt.

Alle Patienten, bei denen der Orthopäde eine axSpA für möglich hielt, wurden - unabhängig vom Befragungs- bzw. Studienergebnis - zum Rheumatologen überwiesen. Die Rheumatologen stellten die Diagnose einer axSpA oder einer anderen Erkrankung (non-axSpA), dokumentierten darüber hinaus für jeden Patienten die ASAS-Klassifikationskriterien, und gaben ggf. eine Therapieempfehlung.

Die von den Orthopäden erfassten Daten (Prüfbogen 1) und die der Rheumatologen (Prüfbogen 2) wurden bis zum 29.02.2016 gesammelt und in einer OpenClinical-Datenbank erfasst. Die Daten wurden von dem statistischen Institut „Biostatistik-Tübingen“ analysiert. Für die statistische Auswertung wurden verschiedene Analysepopulationen gebildet: die Gesamtpopulation (Pop-G), die alle korrekt eingeschlossenen Patienten umfasst; die Population Orthopäde und Rheumatologe (Pop-OR), die alle sowohl vom Orthopäden als auch vom Rheumatologen gesehenen Patienten umfasst, hiervon noch unterschieden Pop-OR-2, für die Pop-OR-Patienten

Tab. 2 Demografische und anamnestische Angaben der axSpA-Patienten $(n=199$

\begin{tabular}{|c|c|c|c|c|c|c|c|c|c|}
\hline \multirow[t]{2}{*}{ Parameter } & \multicolumn{6}{|l|}{$N$} & \multicolumn{3}{|c|}{ Perzentile } \\
\hline & Gültig & Miss & Mw & Std & Min & Max & 25 & Median & 75 \\
\hline \multicolumn{10}{|l|}{ Alter (Jahre) } \\
\hline $\operatorname{axSpA}(n=199)$ & 199 & 0 & 37,7 & 11,1 & 18 & 75 & 29,0 & 36,0 & 46,0 \\
\hline $\mathrm{AS}(n=87)$ & 87 & 0 & 40,6 & 11,8 & 20 & 75 & 31,0 & 40,0 & 51,0 \\
\hline$n r-a x S p A(n=112)$ & 112 & 0 & 35,5 & 10,1 & 18 & 74 & 28,0 & 34,0 & 42,0 \\
\hline \multicolumn{10}{|c|}{ Dauer Rückenschmerz (Monate) } \\
\hline $\operatorname{axSpA}(n=199)$ & 199 & 0 & 98,5 & 105,9 & 3,50 & 600 & 18,0 & 60,0 & 120,0 \\
\hline $\mathrm{AS}(n=87)$ & 87 & 0 & 124,1 & 103,3 & 5,00 & 420 & 36,0 & 109,0 & 204,0 \\
\hline$n r-a x S p A(n=112)$ & 112 & 0 & 78,8 & 104,2 & 3,50 & 600 & 10,0 & 45,0 & 108,0 \\
\hline \multicolumn{10}{|c|}{ Dauer Symptome (Monate) } \\
\hline $\operatorname{axSpA}(n=199)$ & 199 & 0 & 99,6 & 105,1 & 1,00 & 660 & 18,0 & 72,0 & 144,0 \\
\hline AS $(n=87)$ & 87 & 0 & 132,1 & 122,7 & 1,00 & 660 & 35,0 & 96,0 & 204,0 \\
\hline nr-axSpA $(n=112)$ & 112 & 0 & 74,3 & 81,8 & 1,00 & 480 & 10,3 & 48,0 & 120,0 \\
\hline
\end{tabular}

axSpA axiale Spondyloarthritis, AS ankylosierende Spondylitis, $n r$-axSpA nicht-röntgenologische axiale Spondyloarthritis

mit plausibler Diagnose; und die Population, die nur vom Orthopäden (PopO) gesehen wurde. Die Pop-OR-2 wurde noch einmal unterteilt in Pop-OR$\mathrm{S} 1$, bei denen eine Bildgebung vorlag, und Pop-OR-S2, bei denen zusätzlich zur Bildgebung ein HLA-B27-Befund vorlag.

In der deskriptiven Analyse wurden die Parameter innerhalb der Populationen unter Angabe von Fallzahl, Mittelwert, Standardabweichung beschrieben und aufgelistet. Für die AWARE-Parameter erfolgte die Berechnung von Sensitivität und Spezifität zur Vorhersage einer axSpA. Die Patienten wurden je nach Anzahl positiver AWARE-Parameter in 3 Gruppen aufgeteilt: AWARE positiv (mindestens 3 positive Parameter, Gruppe A), AWARE negativ (keine positiven Parameter, Gruppe B), und AWARE unentschlossen (1 oder 2 positive Parameter, Gruppe C).

Mithilfe uni- und multivariater Regressionsanalysen wurden dann Risikofaktoren für das Vorliegen einer axSpA ermittelt. Zudem wurden für die möglichen Identifikationsparameter einer axSpA auch die Likelihood Ratios analysiert.

Für die Studie lag ein positives Ethikvotum der Universität Münster vor.

\section{Ergebnisse}

Insgesamt wurden 1543 durch die Orthopäden dokumentierte Patienten in die Studie konsekutiv eingeschlossen. Hiervon konnten 1306 Patienten (84,6\%) in die Analysepopulation (Pop-G) aufgenommen werden. 237 Patienten (15,4\%) mussten aus verschiedenen Gründen (meist fehlende Parameter) ausgeschlossen werden. Von den 1306 Patienten wurden insgesamt 502 Patienten $(38,4 \%)$ anschließend auch von einem Rheumatologen gesehen (Pop-OR). Für 500 dieser Patienten lag letztlich eine plausible Diagnose vor (Pop-OR-2).

Insgesamt lag für 405 der 500 Patienten $(81,0 \%)$ eine Bildgebung (Röntgen und/oder MRT) vor (Pop-OR-S1). Für 343 der 405 Patienten (84,7\%) lag zusätzlich ein HLA-B27-Befund vor (PopOR-S2).

Das mittlere Alter der gesamten Analysepopulation $(n=1306)$ war $38,5 \pm$ 11,7 Jahre. 49,8\% waren männlich. Die durchschnittliche Dauer der Rückenschmerzen betrug 96,3 $\pm 105,2$ Monate, das sind im Mittel etwa 8 Jahre.

Das mittlere Alter der von Rheumatologen gesehenen und diagnostizierten Patienten $(n=500)$ war mit $38 \pm 11,5$ Jahren sehrähnlich. Dies traf auch aufdie anderen demografischen Daten zu: 46,2\% waren männlich, und die durchschnittliche Dauer der Rückenschmerzen betrug 94,1 \pm 103,6 Monate (•Tab. 1). 
Z Rheumatol 2019·78:568-576 https://doi.org/10.1007/s00393-018-0550-2

(c) Der/die Autor(en) 2018

\section{J. Braun · T. Mosch • I. Fischer · U. Kiltz}

\section{Identifikation von Patienten mit axialer Spondyloarthritis in der Primärversorgung (AWARE-Studie)}

\section{Zusammenfassung}

Hintergrund. Die Früherkennung von Patienten mit axialer Spondyloarthritis (axSpA) in der Primärversorgung ist schwierig. Die Kombination von verschiedenen auf entzündlichen Rückenschmerz hinweisenden Parametern (AWARE-Kriterien) war in einer ersten Studie vorteilhaft: Alter bei Beginn der Rückenschmerzen $<35$ Jahre, Wechselseitiger Gesäßschmerz, Ansprechen auf NSAR innerhalb von $48 \mathrm{~h}$, Regung/Bewegung bringt Besserung, Erwachen in der zweiten Nachthälfte aufgrund dieser Rückenschmerzen

Ziel der Arbeit. Überprüfung der Kriterien zur Identifizierung von jungen Patienten mit axSpA bei chronischem Rückenschmerz ( $\geq 3$ Monate; RS).

Material und Methoden. Bei erwachsenen Patienten mit chronischem RS und einem Alter bei Auftreten der Symptome $<45$ Jahre wurden von den Orthopäden verschiedene mögliche axSpA-Merkmale vor der Überweisung zum Rheumatologen dokumentiert.

Ergebnisse. Insgesamt wurden die Daten von 1306 Patienten erfasst. Von diesen wurden letztlich 500 Patienten auch von Rheumatologen gesehen; bei 199 Patienten (39,8\%) wurde eine axSpA diagnostiziert, während 301 (60,2\%) unspezifische Rückenschmerzen aufwiesen. Insgesamt hatten 87 Patienten eine ankylosierende Spondylitis (44\%) und 112 eine nicht-röntgenologische axSpA (56\%). Die ASAS-Klassifikationskriterien erfüllten 226 Patienten (45,2\%). Das mittlere Alter der axSpA-Patienten war 38 Jahre, $56 \%$ männlich, mittlere Dauer der Rückenschmerzen 98 Monate. Die AWARE-Kriterien hatten eine Sensitivität und Spezifität von 69,3 und $40,3 \%(n=343)$, wenn $\geq 4 / 5$ Kriterien gewählt wurden. Eine positive Bildgebung für Sakroiliitis (Magnetresonanztomographie
[MRT] oder Röntgen) lag bei $77 \%$ und ein positives HLA-B27 bei $59 \%$ der axSpAPatienten vor. Die Kombination von positiver Bildgebung und HLA-B27 hatte die höchste Likelihood Ratio für die Diagnose axSpA. Diskussion. Auch wenn das hier verwendete Studiendesign zu einer Vorselektion und damit zu einer Verzerrung bei der statistischen Auswertung geführt hat, bestätigt die Studie den Nutzen der AWARE-Kriterien zur Früherkennung von Patienten mit axSpA. In weiteren Studien wird aktuell auch der zweistufige Ansatz mit zunächst 3 klinischen Fragen und dann einem fakultativen HLA-B27Test weiter untersucht.

Schlüsselwörter

Axiale Spondyloarthritis · Früherkennung . HLA-B27 - Magnetresonanztomographie . Nichtsteroidale Antiphlogistika

\section{Identification of patients with axial spondylarthritis in primary care (AWARE study)}

\section{Abstract}

Background. Early detection of patients with axial spondylarthritis (axSpA) in primary care is difficult. The combination of various parameters indicative of inflammatory back pain (AWARE criteria) was found to be beneficial in an initial study.

Objective. Review of the criteria for the identification of young patients with axSpA and chronic back pain ( $\geq 3$ months of back pain).

Material and methods. In adult patients with chronic back pain and age at onset of symptoms $<45$ years, orthopedic surgeons documented various possible axSpA characteristics before referral to the rheumatologist.

Results. Overall, the data from 1306 patients were recorded. Of these, ultimately 500 pa- tients were also seen by rheumatologists, 199 patients (39.8\%) were diagnosed with axSpA while 301 (60.2\%) had non-specific back pain. A total of 87 patients had ankylosing spondylitis (44\%) and 112 non-radiographic axSpA (56\%). The ASAS classification criteria were fulfilled by 226 patients (45.2\%). The mean age of axSpA patients was 38 years, $56 \%$ were male with a mean duration of back pain of 98 months. The AWARE criteria had a sensitivity and specificity of $69.3 \%$ and $40.3 \%(n=343)$, respectively, when $\geq 4 / 5$ criteria were chosen. Positive imaging for sacroiliitis using magnetic resonance imaging (MRI) or X-ray was present in $77 \%$ of patients and positive HLA-B27 was identified in $59 \%$ of axSpA patients. The combination of positive imaging and HLA-B27 had the highest likelihood ratio for diagnosis of axSpA.

Conclusion. Although the study design used here led to a preselection and thus to a bias in the statistical evaluation, the study confirmed the benefit of the AWARE criteria for the early detection of patients with axSpA. In further studies, the 2-stage approach with initially 3 clinical questions and then an optional HLA-B27 test is currently being investigated further.

\section{Keywords}

Axial spondylarthritis - Early diagnosis .

Magnetic resonance imaging · HLA B27 · Nonsteroidal anti-inflammatory drugs
Das mittlere Alter der axSpA-Patienten war $37,7 \pm 11,1$ Jahre $(n=199)$, das mittlere Alter der AS-Patienten war $40,6 \pm 11,8$ Jahre $(n=87)$, das mittlere Alter der nr-axSpA-Patienten war $35,5 \pm 10,1$ Jahre $(n=12)$.

$56,3 \%$ der axSpA-Patienten waren männlich $(62,1 \%$ der AS-Patienten
[ $n=54]$ und 51,8\% der nr-axSpA-Patienten $[n=58])$.

Die durchschnittliche Dauer der Rückenschmerzen der axSpA-Patienten betrug 98,5 \pm 105,9 Monate (im Mittel etwa 8 Jahre). Die durchschnittliche Dauer der Rückenschmerzen der AS-Patienten betrug 124,1 \pm 103,3 Monate (im Mittel etwa 10 Jahre). Die durchschnittli- che Dauer der Rückenschmerzen der nraxSpA-Patienten betrug 78,8 \pm 104,2 Monate (im Mittel etwa 6,5 Jahre) (• Tab. 2 und 3 ).

Die AWARE-Parameter waren in der Gesamtpopulation (Pop-G) und in der von den Rheumatologen diagnostizierten Population (Pop-OR-2 sowie PopOR-S2) relativ gleich verteilt (• Tab. 4). 
Tab. 3 Geschlechtsverteilung bei Patienten mit axialer Spondyloarthritis

\begin{tabular}{|llllll}
\hline Parameter [\%(n)] & Ausprägung & \multicolumn{4}{|c}{ Population mit axSpA (n= 199) } \\
& & $\begin{array}{l}\text { Gesamt } \\
(\boldsymbol{n}=199)\end{array}$ & $\begin{array}{l}\text { AS } \\
(\boldsymbol{n}=\mathbf{8 7})\end{array}$ & $\begin{array}{l}\text { nr-axSpA } \\
(\boldsymbol{n}=112)\end{array}$ \\
\hline Geschlecht & Männlich & $56,3(112)$ & $62,1(54)$ & $51,8(58)$ \\
& Weiblich & $43,7(87)$ & $37,9(33)$ & $48,2(54)$
\end{tabular}

AS ankylosierende Spondylitis, axSpA axiale Spondyloarthritis, $n r-a x S p A$ nicht-röntgenologische axiale Spondyloarthritis

\begin{tabular}{|c|c|c|c|c|c|}
\hline $\begin{array}{l}\text { AWARE-Parameter } \\
{[\%(n)]}\end{array}$ & $\begin{array}{l}\text { Pop-G } \\
(n=1306)\end{array}$ & $\begin{array}{l}\text { Pop-0 } \\
(n=797)\end{array}$ & $\begin{array}{l}\text { Pop-OR-2 } \\
(n=500)\end{array}$ & $\begin{array}{l}\text { Pop-OR } \\
(n=502)\end{array}$ & $\begin{array}{l}\text { Pop-OR-S2 }{ }^{a} \\
(n=343)\end{array}$ \\
\hline $\begin{array}{l}\text { Alter bei Beginn der } \\
\text { Rückenschmerzen } \\
<35 \text { Jahre }\end{array}$ & $81,2(1060)$ & $81,9(653)$ & $80,0(400)$ & $80,1(402)$ & $82,8(284)$ \\
\hline $\begin{array}{l}\text { Wechselseitiger Gesäß- } \\
\text { schmerz }\end{array}$ & $69,4(907)$ & $68,1(543)$ & 71,4 (357) & 71,5 (359) & 72,6 (249) \\
\hline $\begin{array}{l}\text { Ansprechen auf NSAR } \\
\text { innerhalb von } 48 \mathrm{~h}\end{array}$ & $66,3(866)$ & $67,1(535)$ & $65,2(326)$ & $65,1(327)$ & $65,6(225)$ \\
\hline $\begin{array}{l}\text { Regung/Bewegung } \\
\text { bringt Besserung }\end{array}$ & $78,9(1030)$ & 79,9 (637) & 77,0 (385) & 77,1 (387) & $76,4(262)$ \\
\hline $\begin{array}{l}\text { Erwachen in der zweiten } \\
\text { Nachthälfte aufgrund } \\
\text { dieser Rückenschmerzen }\end{array}$ & 74,8 (977) & 72,4 (577) & 78,4 (392) & 78,3 (393) & 80,5 (276) \\
\hline $\begin{array}{l}\text { Keiner dieser Parameter } \\
\text { trifft zu }\end{array}$ & $0,5(7)$ & $0,5(4)$ & $0,6(3)$ & $0,6(3)$ & $0,3(1)$ \\
\hline
\end{tabular}

Tab. 5 Diagnosen des Rheumatologen

\begin{tabular}{|c|c|c|}
\hline Parameter $[\%(n)]$ & Pop-OR-2 $(n=500)$ & $\begin{array}{l}\text { Pop-OR-S2 }^{a} \\
(n=343)\end{array}$ \\
\hline Ankylosierende Spondylitis (AS) & $17,4(87)$ & $19,2(66)$ \\
\hline Nicht-röntgenologische axiale SpA (nr-axSpA) & $22,4(112)$ & $20,7(71)$ \\
\hline Axiale SpA (gesamt) & $39,8(199)$ & $39,9(137)$ \\
\hline Non-axSpA ${ }^{b}$ & $60,2(301)$ & $60,1(206)$ \\
\hline Mechanischer Rückenschmerz & $52,2(261)$ & $51,6(177)$ \\
\hline Andere Diagnosen & $8,0(40)$ & $8,5(29)$ \\
\hline
\end{tabular}

Tab. 6 Sensitivität und Spezifität der AWARE-Parameter $(n=343)$

\begin{tabular}{|lll|}
\hline AWARE-Parameter & $\begin{array}{l}\text { Sensitivität } \\
{[\%, 95 \%-\mathbf{K I}]}\end{array}$ & $\begin{array}{l}\text { Spezifität } \\
{[\%, \mathbf{9 5} \%-\mathbf{K I}]}\end{array}$ \\
\hline Alter bei Beginn der Rückenschmerzen <35 Jahre & $81,8(74,3-87,8)$ & $16,5(11,7-22,3)$ \\
\hline Wechselseitiger Gesäßschmerz & $78,8(71,0-85,3)$ & $31,6(25,3-38,4)$ \\
\hline Ansprechen auf NSAR innerhalb von 48 h & $73,7(65,5-80,9)$ & $39,8(33,1-46,8)$ \\
\hline $\begin{array}{l}\text { Regung/Bewegung bringt Besserung } \\
\text { Erwachen in der zweiten Nachthälfte aufgrund dieser }\end{array}$ & $79,6(71,8-86,0)$ & $25,7(19,9-32,3)$ \\
Rückenschmerzen & $81,8(74,3-87,8)$ & $20,4(15,1-26,5)$ \\
\hline NSAR nicht-steroidale Antirheumatika & & \\
\hline
\end{tabular}

Insgesamt wurde bei 199 Patienten $(39,8 \%)$ die Diagnose einer axSpA gestellt, 301 Patienten $(60,2 \%)$ hatten mechanische Rückenschmerzen oder andere Diagnosen (non-axSpA) (• Tab. 5).

Von den 199 Patienten mit axSpA hatten 87 Patienten eine AS (43,7\% der 199 bzw. 17,4\% der 500 Patienten) und 112 Patienten eine nr-axSpA (56,3\% der 199 bzw. 22,4\% der 500 Patienten).

HLA-B27 wurde bei insgesamt 162 von 343 Patienten positiv getestet (47,2\% von 343 bzw. $32,4 \%$ von 500). Bei $157 \mathrm{~Pa}$ tienten von 500 Patienten lag kein Testergebnis vor (31,4\%). Insgesamt waren 117 der 199 axSpA-Patienten (58,8\%) und 45 der 301 Non-axSpA-Patienten (15\%) HLA-B27-positiv.

Von den 405 Fällen, bei denen eine Bildgebung (MRT und/oder Röntgen) vorlag, zeigte sich nach Ansicht der Rheumatologen bei 170 Fällen eine Sakroiliitis (42\% bzw. 34,0\% von 500). Bei $154 \mathrm{der}$ 199 Patienten mit axSpA wurde die Bildgebung als positiv bewertet $(77,4 \%)$. Im Vergleich war dies nur bei 16 der $206 \mathrm{~Pa}$ tienten (mit Bildgebung) mit non-axSpA der Fall (7,8\%).

Insgesamt erfüllten 226 Patienten der 500 von Rheumatologen gesehenen $\mathrm{Pa}$ tienten die ASAS-Klassifikationskriterien $(45,2 \%)$. Von den 199 axSpA-Patienten erfüllten 179 Patienten (89,9\%) die ASAS-Klassifikationskriterien. Hiervon hatten 84 Patienten eine AS (46,9\%) und 95 Patienten eine nr-axSpA (53,1\%). Von den 301 Non-axSpA-Patienten erfüllten 47 die ASAS-Klassifikationskriterien $(15,6 \%)$.

Zur Darstellung der diagnostischen Gütekriterien werden im Folgenden nur die Daten der 343 Patienten gezeigt, bei denen sowohl Ergebnisse der Bildgebung als auch der HLA-B27-Befund vorlagen (68,6\% der insgesamt rheumatologisch gesehenen Patienten).

Die Sensitivität und Spezifität der AWARE-Parameter für die Vorhersage einer axSpA sind in $\bullet$ Tab. 6 und 7 dargestellt. Die AWARE-Parameter hatten eine Sensitivität und Spezifität für eine axSpA von 93,4 und $14,6 \%$, wenn $\geq 3$ Parameter zutrafen, und von 6,57 und $85,4 \%$ wenn <3 Parameter zutrafen (• Tab. 8).

Die Verteilung der AWARE-Parameter bei AS und nr-axSpA war ähnlich 
Tab. 7 Sensitivität und Spezifität der Anzahl positiver AWARE-Parameter $(n=343)$

\begin{tabular}{l|l|l}
\hline AWARE-Parameter & $\begin{array}{l}\text { Sensitivität } \\
{[\%, 95 \%-K I]}\end{array}$ & $\begin{array}{l}\text { Spezifität } \\
{[\%, 95 \%-K I]}\end{array}$ \\
\hline 1 trifft zu & $100(97,3-100)$ & $0,97(0,12-3,46)$ \\
\hline 2 treffen zu & $99,3(96,0-100)$ & $1,94(0,53-4,90)$ \\
\hline 3 treffen zu & $93,4(97,9-97,0)$ & $14,6(10,1-20,1)$ \\
\hline 4 treffen zu & $69,3(60,9-76,9)$ & $40,3(33,5-47,3)$ \\
\hline Alle 5 treffen zu & $33,6(25,7-42,1)$ & $76,2(69,8-81,9)$ \\
\hline Kein AWARE-Parameter trifft zu & $0(0-2,66)$ & $99,0(96,5-99,9)$
\end{tabular}

Tab. 8 Sensitivität und Spezifität der AWARE-Gruppen $(n=343)$

\begin{tabular}{l|l|l}
\hline AWARE-Parameter & $\begin{array}{l}\text { Sensitivität } \\
{[\%, 95 \%-K I]}\end{array}$ & $\begin{array}{l}\text { Spezifität } \\
{[\%, 95 \%-K I]}\end{array}$ \\
\hline Gruppe A (AWARE positiv, $n=304)$ & $93,4(87,9-97,0)$ & $14,6(10,1-20,1)$ \\
\hline Gruppe B (AWARE negativ, $n=2)$ & $0(0-2,66)$ & $99,0(96,5-99,9)$ \\
\hline Gruppe C (AWARE unentschlossen, $n=37)$ & $6,57(3,05-12,1)$ & $86,4(81,0-91,0)$ \\
\hline Gruppe D (AWARE <3 pos., $n=39)$ & $6,57(3,05-12,1)$ & $85,4(79,9-90,0)$ \\
Gruppe F (AWARE $\geq 4$ pos., $n=218)$ & $69,3(60,9-76,9)$ & $40,3(33,5-47,3)$ \\
Gruppe H (AWARE $\geq 2$ pos., $n=338)$ & $99,3(96,0-100)$ & $1,94(0,53-4,90)$
\end{tabular}

\begin{tabular}{|c|c|c|c|c|c|}
\hline \multirow[t]{2}{*}{$\begin{array}{l}\text { Parameter ( } n=343 \text {; angegeben ist } \\
\text { die Risikogruppe) }\end{array}$} & \multicolumn{2}{|c|}{$\begin{array}{l}\text { Univariate } \\
\text { Analyse }\end{array}$} & \multicolumn{3}{|c|}{$\begin{array}{l}\text { Multivariate Analyse }(n=343) \\
\text { volles Modell }\end{array}$} \\
\hline & $\mathbf{R R}$ & $p$-Wert & OR & $95 \%-K I$ & $p$-Wert \\
\hline $\begin{array}{l}\text { Alter bei Beginn der Rückenschmerzen } \\
<35 \text { Jahre }\end{array}$ & 0,886 & 0,675 & 0,916 & $0,513-1,634$ & 0,766 \\
\hline Wechselseitiger Gesäßschmerz & 1,717 & 0,036 & 1,638 & $0,983-2,728$ & 0,058 \\
\hline $\begin{array}{l}\text { Ansprechen auf NSAR innerhalb von } \\
48 \mathrm{~h}\end{array}$ & 1,855 & 0,010 & 1,759 & $1,089-2,843$ & 0,021 \\
\hline Regung/Bewegung bringt Besserung & 1,349 & 0,259 & 1,194 & $0,697-2,045$ & 0,519 \\
\hline $\begin{array}{l}\text { Erwachen in der zweiten Nachthälfte } \\
\text { aufgrund dieser Rückenschmerzen }\end{array}$ & 1,147 & 0,625 & 1,093 & $0,621-1,924$ & 0,758 \\
\hline Psoriasis und/oder CED ${ }^{a}$ & 1,352 & 0,432 & - & - & - \\
\hline
\end{tabular}

Tab. 10 Optimiertes Endmodell

\begin{tabular}{l|lll} 
Parameter & \multicolumn{3}{l}{ Multivariate Analyse $(\boldsymbol{n}=\mathbf{3 4 3})$} \\
& OR & $\mathbf{9 5 \% - K \mathbf { l }}$ & $\boldsymbol{p}$-Wert \\
\hline Wechselseitiger Gesäßschmerz & 1,662 & $0,993-2,748$ & 0,053 \\
\hline Ansprechen auf NSAR innerhalb von 48 h & 1,802 & $1,121-2,898$ & 0,015
\end{tabular}

(nicht gezeigt). Wechselseitiger Gesäßschmerz und schmerzbedingtes Erwachen in der zweiten Nachthälfte war bei Patienten mit AS etwas häufiger; Ansprechen auf NSAR innerhalb von $48 \mathrm{~h}$ und Besserung bei Bewegung war bei Patienten mit nr-axSpA etwas häufiger.

Logistische Regressionsanalysen $\mathrm{zu}$ Risikofaktoren für eine axSpA ergaben ein 1,8-fach höheres Risiko für eine der Identifikationsparameter für die beiden Gruppen axSpA und non-axSpA ist in - Tab. 11 dargestellt.

Die Bildgebung (MRT und/oder Röntgen) war bei insgesamt 134 von $343 \mathrm{~Pa}$ tienten $(39,1 \%)$ positiv. $91 \%$ dieser $\mathrm{Pa}$ tienten hatten eine axSpA vs. $9 \%$ hatten keine axSpA. Insgesamt hatten 122 der 141 axSpA-Patienten (86,5\%) eine als positiv gewertete Bildgebung vs. 12 der 202 Non-axSpA-Patienten (5,9\%). HLA-B27 war bei insgesamt 140 von 343 Patienten $(40,8 \%)$ positiv. $70 \%$ dieser Patienten hatten eine axSpA vs. $30 \%$ eine nonaxSpA. Insgesamt waren 98 von 141 axSpA-Patienten (69,5\%) HLA-B27 positiv vs. 42 von 202 Non-axSpA-Patienten (20,8\%).

Bei den AWARE-Parametern sah die Verteilung so aus: $82,3 \%$ der axSpA-Patienten hatte ein Alter bei Beschwerdebeginn $<35$ Jahre vs. 83,1 \% der Non-axSpA-Patienten. Wechselseitigen Gesäßschmerz hatten 78,7\% der axSpA-Patienten vs. $68,3 \%$ der Non-axSpA-Patienten. Ansprechen auf NSAR innerhalb von $48 \mathrm{~h}$ gaben $72,3 \%$ der axSpA-Patienten vs. $61 \%$ der Non-axSpA-Patienten an. Besserung der Rückenschmerzen bei Bewegung lag bei $78,7 \%$ vs. $75 \%$ der NonaxSpA-Patienten vor, Aufwachen in der zweiten Nachthälfte schmerzbedingt bei $82,3 \%$ vs. $79,2 \%$.

$62,4 \%$ der axSpA-Patienten vs. 30,7 \% der Non-axSpA-Patienten sprachen gut auf NSAR an, und $29,8 \%$ vs. $7,9 \%$ hatten ein erhöhtes CRP. Entzündlicher Rückenschmerz wurde von $76,6 \%$ vs. $28,7 \%$ angegeben. Die Familienanamnese war bei 10,6\% der axSpA-Patienten vs. $4,5 \%$ der Non-axSpA-Patienten positiv. Nur eine Minderheit der axSpAPatienten hatte eine Arthritis (7,8\%, vs. $3,5 \%$ der Non-axSpA-Patienten), Enthesitis $(8,5 \%$ vs. $5 \%$ der Non-axSpAPatienten), Daktylitis (1,4\% vs. $0,5 \%$ der Non-axSpA-Patienten), Uveitis (5,7\% vs. $1 \%$ der Non-axSpA-Patienten), Psoriasis $(5,7 \%$ vs. $3,5 \%$ der Non-axSpAPatienten) oder CED (5\% vs. $2 \%$ der Non-axSpA-Patienten).

Der Nachweis einer Sakroiliitis (MRT oder Röntgen) ergab als einzelner Identifikationsparameter die höchste positive Likelihood Ratio (LR+) für die Diagnose einer axSpA $(14,6)$. Die LR+ war bei der 
Tab. 11 Verteilung der Identifikationsparameter für eine axiale SpA $(n=343)$

Parameter [\% (n)]

Ausprägung

Pop-OR-2 ${ }^{\mathrm{a}}$

$(n=343)$

\begin{tabular}{llll} 
& & $\begin{array}{l}\text { axSpA } \\
(\boldsymbol{n}=\mathbf{1 4 1})\end{array}$ & $\begin{array}{l}\text { Non-axSpA } \\
(\boldsymbol{n}=\mathbf{2 0 2})\end{array}$ \\
\hline Bildgebung positiv & Ja $(n=134)$ & $91,0(122)$ & $9,0(12)$ \\
& Nein $(n=209)$ & $9,1(19)$ & $90,9(190)$ \\
& Gesamt & $86,5 \%$ & $5,9 \%$ \\
\hline HLA-B27-positiv & Ja $(n=140)$ & $70,0(98)$ & $30,0(42)$ \\
& Nein $(n=203)$ & $21,2(43)$ & $78,8(160)$ \\
& Gesamt & $69,5 \%$ & $20,8 \%$
\end{tabular}

AWARE-Parameter

Alter bei Beginn der Rückenschmerzen <35 J

Alter bei Beginn der Rückenschmerzen $<35 \mathrm{~J}$

Wechselseitiger Gesäßschmerz

Ja $(n=284)$

$40,8(116)$

$59,2(168)$

Nein $(n=59)$

$42,4(25)$

$57,6(34)$

Gesamt

Ja $(n=249)$

$82,8 \%$

$83,1 \%$

Nein $(n=94)$

$44,6(111)$

$55,4(138)$

Gesamt

$31,9(30)$

$68,1(64)$

Ansprechen auf NSAR innerhalb von $48 \mathrm{~h}$

Ja $(n=225)$

$78,7 \%$

$68,3 \%$

Nein $(n=118)$

$54,7(123)$

Gesamt

33,1 (39)

$66,9(79)$

Regung/Bewegung bringt Besserung

Ja $(n=262)$

$72,3 \%$

$61 \%$

Nein $(n=81)$

$42,4(111)$

$57,6(151)$

Gesamt

$37,0(30)$

$63,0(51)$

Erwachen in der zweiten Nachthälfte wegen der Rückenschmerzen

SpA-Parameter

Gutes Ansprechen auf NSAR

Erhöhtes CRP

Arthritis

Enthesitis (Ferse)

Uveitis

Daktylitis

Psoriasis

$\begin{array}{lll}\text { Ja }(n=276) & 42,0(116) & 58,0(160) \\ \text { Nein }(n=67) & 37,3(25) & 62,7(42) \\ \text { Gesamt } & 82,3 \% & 79,2 \%\end{array}$

\begin{tabular}{|l|l|l}
\hline Ja $(n=150)$ & $58,7(88)$ & $41,3(62)$ \\
\hline Nein $(n=193)$ & $27,5(53)$ & $72,5(140)$ \\
\hline Gesamt & $62,4 \%$ & $30,7 \%$ \\
\hline Ja $(n=58)$ & $72,4(42)$ & $27,6(16)$ \\
\hline Nein $(n=285)$ & $34,7(99)$ & $65,3(186)$ \\
\hline Gesamt & $29,8 \%$ & $7,9 \%$ \\
\hline Ja $(n=18)$ & $61,1(11)$ & $38,9(7)$ \\
\hline Nein $(n=325)$ & $40,0(130)$ & $60,0(195)$ \\
\hline Gesamt & $7,8 \%$ & $3,5 \%$ \\
\hline Ja $(n=22)$ & $54,5(12)$ & $45,5(10)$ \\
\hline Nein $(n=321)$ & $40,2(129)$ & $59,8(192)$ \\
\hline Gesamt & $8,5 \%$ & $5 \%$ \\
\hline Ja $(n=10)$ & $80,0(8)$ & $20,0(2)$ \\
\hline Nein $(n=333)$ & $39,9(133)$ & $60,1(200)$ \\
\hline Gesamt & $5,7 \%$ & $1 \%$ \\
\hline Ja $(n=3)$ & $66,7(2)$ & $33,3(1)$ \\
\hline Nein $(n=340)$ & $40,9(139)$ & $59,1(201)$ \\
\hline Gesamt & $1,4 \%$ & $0,5 \%$ \\
\hline Ja $(n=15)$ & $53,3(8)$ & $46,7(7)$ \\
\hline Nein $(n=328)$ & $40,5(133)$ & $59,5(195)$ \\
\hline Gesamt & $5,7 \%$ & $3,5 \%$ \\
\hline & & \\
\hline
\end{tabular}

Kombination von Identifikationsparametern bei positiver Bildgebung und positiver HLA-B27-Diagnostik am höchsten $(120,3)$, dies jedoch auf Kosten der negativen Likelihood Ratio (• Tab. 12).

\section{Diskussion}

Obwohl das Studiendesign zur Vorselektion der Patienten und damit zu einem Selektionsbias für die statistische Analyse geführt hat, unterstreicht diese Studie den Nutzen der ursprünglichen AWARE-Parameter zur Identifikation von jungen Patienten mit chronischen Rückenschmerzen in der Primärversorgung, da insgesamt etwa $40 \%$ der zum Rheumatologen überwiesenen $\mathrm{Pa}$ tienten, dort als axSpA identifiziert und diagnostiziert wurden. Der Selektionsbias kam dadurch zustande, dass den Orthopäden das gute Abschneiden der AWARE-Parameter in der Pilotstudie bekannt war und dass sich damit ein deutlicher Trend ergab, Patienten als AWAREpositiv auszuweisen und diese bevorzugt zu überweisen. Dies hatte zur Folge, dass die „Kontrollgruppe“ der axSpA-Gruppe formal zu ähnlich war. Für diese Interpretation sprechen zum einen die Daten selbst und zum anderen der hervorstechende Unterschied zwischen den von den Rheumatologen nicht überprüften AWARE-Parametern und der rheumatologischen Einschätzung des entzündlichen Rückenschmerzes (bei 76,6\% bei axSpA-vs. $28,7 \%$ bei Non-axSpA-Patienten). In dem Zusammenhang ist auch das Ergebnis der ASAS-Klassifikationskriterien von Interesse: Diese wurden, sehr ähnlich zur Originalpublikation [24], von $89,9 \%$ der axSpA-Patienten, aber auch von $15,6 \%$ der Non-axSpA-Patienten erfüllt; dies entspricht der bisher in den meisten Studien gefundenen Sensitivität und Spezifität.

Die wichtige Rolle der Bildgebung und des HLA-B27 wird durch diese Studie bestätigt. Diese Kombination (positive Bildgebung und HLA-B27+) sowie die Kombination mit entzündlichem Rückenschmerz hatte entsprechend die höchste Likelihood Ratio für das Vorliegen der Diagnose axSpA ergeben.

Wie bereits in früheren Studien $[9,10$, $13,17,21,27]$ gezeigt, funktionieren die 


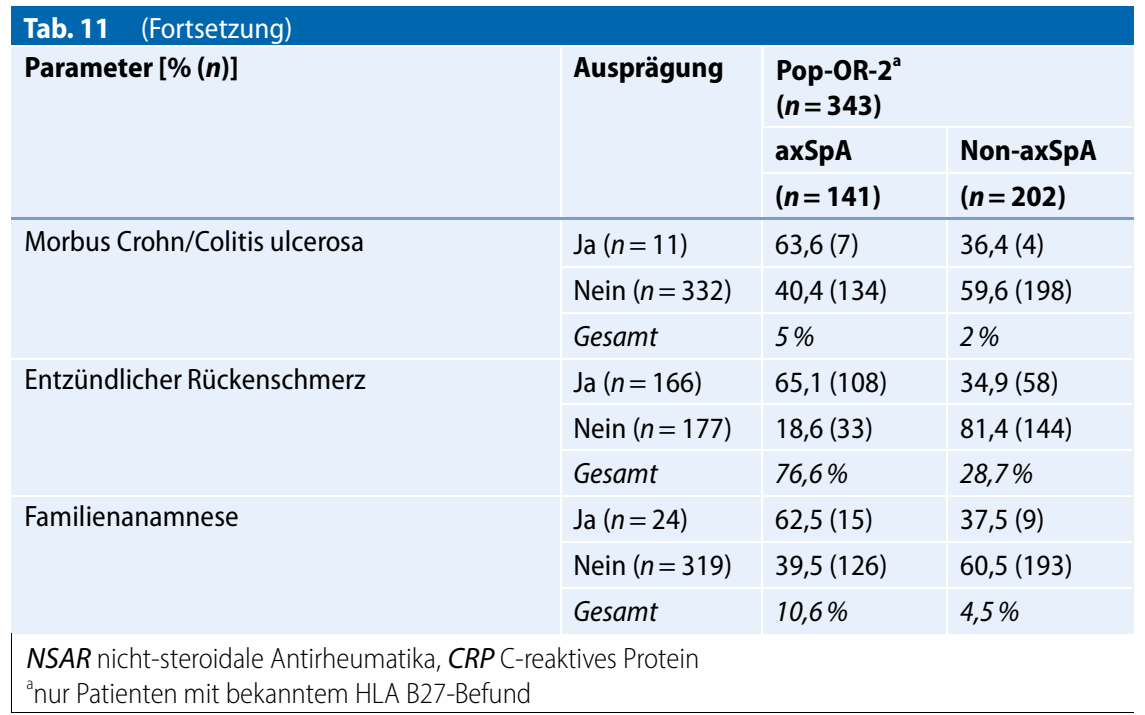

\begin{tabular}{|c|c|c|c|c|}
\hline Identifikationsparameter & $\begin{array}{l}\text { Sensitivität } \\
{[\%, 95 \%-K I]}\end{array}$ & $\begin{array}{l}\text { Spezifität } \\
{[\%, 95 \%-K I]}\end{array}$ & LR+ & LR- \\
\hline Bildgebung positiv & $86,5(79,8-91,7)$ & $94,1(89,9-96,9)$ & 14,6 & 0,143 \\
\hline HLA-B27-Diagnostik positiv & $69,5(61,2-77,0)$ & $79,2(73,0-84,6)$ & 3,34 & 0,385 \\
\hline Entzündlicher Rückenschmerz & $76,6(68,7-83,3)$ & $71,3(64,5-77,4)$ & 2,67 & 0,328 \\
\hline $\begin{array}{l}\text { Bildgebung positiv und ent- } \\
\text { zündlicher Rückenschmerz }\end{array}$ & $66,0(57,5-73,7)$ & $97,0(93,7-98,9)$ & 22,2 & 0,351 \\
\hline $\begin{array}{l}\text { Bildgebung positiv und } \\
\text { HLA-B27-Diagnostik positiv }\end{array}$ & $59,6(51,0-67,8)$ & $99,5(97,3-100)$ & 120,3 & 0,406 \\
\hline $\begin{array}{l}\text { Bildgebung positiv, entzünd- } \\
\text { licher Rückenschmerz und } \\
\text { HLA-B27-Diagnostik positiv }\end{array}$ & $48,2(39,7-56,8)$ & $99,5(97,3-100)$ & 97,4 & 0,520 \\
\hline LR Likelihood ratio & & & & \\
\hline
\end{tabular}

Eingangskriterien Chronizität (>3 Monate) und früher Beginn ( $<45$ Jahre) wahrscheinlich schon ziemlich gut zur Vorselektion von Patienten mit axSpA.

Ein optimales Zuweisungssystem ist aus mehreren Gründen wichtig: (i) Zum einen kann der Rheumatologe aus Kapazitätsgründen nicht mehr als 2 bis 3 Patienten mit chronischem Rückenschmerz sehen [31], um bei einem Patienten die Diagnose axSpA zu sichern, und (ii) zum anderen ist ein solches für die Erreichung von etablierten Versorgungszielen [15] und v. a. die Früherkennung notwendig. Dazu gehört neben der möglichst zeitnahen Diagnosesicherung auch der frühe Einsatz einer effektiven antientzündlichen Therapie z. B. mit nichtsteroidalen Antiphlogistika [3]. Auch in dieser Studie zeigte sich die diskriminatorische Kapazität dieser Substanzen für die Diagnosestellung der axSpA [1]. Durch den Einsatz
Stimmigkeit der AWARE-Fragen vorgesehen.

In laufenden Studien wird jetzt die etwas später entwickelte ebenfalls datenbasierte Zwei-Phasen-Strategie zur Identifikation von axSpA-Patienten [10] weiter untersucht. Diese beinhaltet zunächst die Klärung von 3 klinischen Fragen (beidseitige Gesäßschmerzen, Besserung bei Bewegung und Psoriasis) und anschließend, falls notwendig, die HLA-B27-Bestimmung. Mit diesem abgestuften Modell könnten wahrscheinlich erhebliche Kosten eingespart werden, da das Risikogen HLA-B27 nicht bei allen Patienten mit Rückenschmerzen bestimmt werden muss.

\section{Fazit für die Praxis}

Um die Früherkennung von Patienten mit axSpA in der Primärversorgung zu verbessern, sind einfache Facharztüberweisungssysteme erforderlich. Die initial wichtigsten Parameter sind zunächst der frühe Beginn und die Chronizität (>3 Monate). In dieser Studie sollten die in einer früheren Studie identifizierten AWARE-Kriterien („,Alter bei Beginn der Rückenschmerzen <35 Jahre", "Wechselseitiger Gesäßschmerz", „Ansprechen auf nichtsteroidale Antirheumatika [NSAR] innerhalb von $48 \mathrm{~h}^{\prime \prime}$, "Regung/Bewegung bringt Besserung", „Erwachen in der zweiten Nachthälfte aufgrund der Rückenschmerzen“) evaluiert werden. Dies gelang nur zum Teil, da in der Kontrollgruppe auch viele Parameter positiv angegeben worden waren. Das Grundkonzept der Herangehensweise hat aber wie schon in früheren Studien funktioniert, denn bei fast $\mathbf{4 0} \%$ der von Rheumatologen gesehenen Patienten wurde eine axSpA diagnostiziert.

\section{Korrespondenzadresse}

\section{Prof. Dr. J. Braun}

Rheumazentrum Ruhrgebiet

Claudiusstr. 45, 44649 Herne, Deutschland

j.braun@rheumazentrum-ruhrgebiet.de

Unterschied zwischen den Gruppen), divergierte die Einschätzung des Rheumatologen hinsichtlich des Vorliegens von entzündlichem Rückenschmerz deutlich. Im Studienprotokoll war keine dezidierte rheumatologische Überprüfung der
Danksagung. Wir danken Frau Dr. med. Annalina Avram für ihre wertvolle Hilfe bei der Herstellung des Manuskripts. 
Förderung. Die Studie wurde von AbbVie finanziert und AbbVie beteiligte sich an der Erstellung des Studiendesigns, der Datenerhebung und Datenauswertung. AbbVie finanzierte die Anfertigung des Manuskripts und war an der Erstellung und Überprüfung beteiligt.

\section{Einhaltung ethischer Richtlinien}

Interessenkonflikt. J. Braun gibt an, dass er die Firma beraten, Vorträge gehalten und dafür Honorare bekommen hat. T. Mosch ist Mitarbeiter der Firma AbbVie und besitzt möglicherweise AbbVie-Aktien. I. Fischer gibt an, dass sie die statistischen Analysen im Auftrag der Firma AbbVie gegen Honorar durchgeführt hat. U. Kiltz gibtan, dass sie die Firma beraten, Vorträge gehalten und dafür Honorare bekommen hat.

\section{Alle beschriebenen Untersuchungen am Menschen} wurden mit Zustimmung der zuständigen EthikKommission (Universität Münster), im Einklang mit nationalem Recht sowie gemäß der Deklaration von Helsinki von 1975 (in der aktuellen, überarbeiteten Fassung) durchgeführt. Von allen beteiligten Patienten liegt eine Einverständniserklärung vor.

Open Access. Dieser Artikel wird unter der Creative Commons Namensnennung 4.0 International Lizenz (http://creativecommons.org/licenses/by/4.0/deed. de) veröffentlicht, welche die Nutzung, Vervielfältigung, Bearbeitung, Verbreitung und Wiedergabe in jeglichem Medium und Format erlaubt, sofern Sie den/die ursprünglichen Autor(en) und die Quelle ordnungsgemäß nennen, einen Link zur Creative Commons Lizenz beifügen und angeben, ob Änderungen vorgenommen wurden.

\section{Literatur}

1. Amor B et al (1995) Are classification criteria for spondylarthropathy useful as diagnostic criteria? Rev RhumEnglEd 62(1):10-15

2. Poddubnyy D, van Tubergen A, LandewéR, Sieper J, van der Heijde D, Assessment of SpondyloArthritis international Society (ASAS) (2015) Defining an optimal referral strategy for patients with a suspicion of axial spondyloarthritis: what is really important? Ann Rheum Dis 74(12):e69 (Response to: 'Evaluating the ASAS recommendations for early referral of axial spondyloarthritis in patients with chronic low back pain; is one parameter present sufficient for primary care practice?' by van Hoeven etal.)

3. Baraliakos X, Kiltz U, Peters S, Appel H, Dybowski F, Igelmann M, Kalthoff L, Krause D, Menne HJ, Saracbasi-Zender E, Schmitz-Bortz E, Vigneswaran M, Braun J (2017) Efficiency of treatment with non-steroidal anti-inflammatory drugs according to current recommendations in patients with radiographic and non-radiographic axial spondyloarthritis. Rheumatology 56(1):95-102

4. Barkham N, Keen HI, Coates LC, O'Connor P, Hensor E, Fraser AD, Cawkwell LS, Bennett A, McGonagle D, Emery P (2009) Clinical and imaging efficacy of infliximab in HLA-B27-Positive patients with magnetic resonance imaging-determined early sacroiliitis. Arthritis Rheum 60(4):946-954

5. Berg Rvan den etal (2013) ASAS modification of the Berlin algorithm for diagnosing axial spondyloarthritis: results from the SPondyloArthritis Caught Early (SPACE)-cohort and from the Assessment of
SpondyloArthritis international Society (ASAS)cohort. Ann Rheum Dis 72:1646-1653

6. Braun J, Inman R (2010) Clinical significance of inflammatory back pain for diagnosis and screening of patients with axial spondyloarthritis. Ann Rheum Dis 69(7):1264-1268

7. Braun J, Kiltz U (2017) Spondyloarthropathies: How should axial spondyloarthritis be diagnosed? Nat Rev Rheumatol 13(5):264-266

8. Braun J, Sieper J (2007) Ankylosing spondylitis. Lancet 369:1379-1390

9. Braun A, Saracbasi E, Grifka J, Schnitker J, Braun J (2011) Identifying patients with axial spondyloarthritis in primary care: how useful are items indicative of inflammatory back pain? Ann Rheum Dis 70(10):1782-1787

10. Braun A, Gnann H, Saracbasi E, Grifka J, Kiltz U, Letschert K, Braun J (2013) Optimizing the identification of patients with axial spondyloarthritis in primary care - the case for a two-step strategy combining the most relevant clinical items with HLA B27. Rheumatology 52(8):1418-1424

11. Braun J, Baraliakos X, Kiltz U, Heldmann F, Sieper $J$ (2015) Classification and diagnosis of axial spondyloarthritis - what is the clinically relevant difference? JRheumatol 42:31-38

12. Bruin F de et al (2016) Prevalence of degenerative changes of the spine on magnetic resonance images and radiographs in patients aged 16-45 years with chronic back pain of short duration in the Spondyloarthritis Caught Early (SPACE) cohort Rheumatology 55:56-65

13. Deodhar A, Strand V, Kay J, Braun J (2016) The term 'non-radiographic axial spondyloarthritis' is much more important to classify than to diagnose patients with axial spondyloarthritis. Ann Rheum Dis 75(5):791-794

14. Ez-Zaitouni Z et al (2017) Presence of multiple spondyloarthritis (SpA) features is important but not sufficient for a diagnosis of axial spondyloarthritis: data from the SPondyloArthritis Caught Early (SPACE) cohort. Ann Rheum Dis 76(6):1086-1092

15. Heijde $D$ van der, Ramiro $S$, Landewé $R$, Baraliakos $X$, van den Bosch F, Sepriano A, Regel A, Ciurea $A$, Dagfinrud H, Dougados M, van Gaalen F, Géher $P$, van der Horst-Bruinsma I, Inman RD, Jongkees M, Kiltz U, Kvien TK, Machado PM, Marzo-Ortega $\mathrm{H}$, Molto A, Navarro-Compàn V, Ozgocmen S, Pimentel-Santos FM, Reveille J, Rudwaleit M, Sieper J, Sampaio-Barros P, Wiek D, Braun J (2017) 2016 update of the ASAS-EULAR management recommendations for axial spondyloarthritis. Ann Rheum Dis 76(6):978-991

16. Heijde D van der, Baraliakos X, Hermann KA, Landewé RBM, Machado PM, Maksymowych WP, Davies OR, de Peyrecave N, Hoepken B, Bauer L, Nurminen T, Braun J (2018) Limited radiographic progression and sustained reductions in MRI inflammation in patients with axial spondyloarthritis: 4-year imaging outcomes from the RAPID-axSpA phase III randomised trial. Ann Rheum Dis 77(5):699-705

17. Hermann J, Giessauf $H$, Schaffler $G$, Ofner $P$ Graninger W (2009) Early spondyloarthritis: usefulness of clinical screening. Rheumatology 48(7):812-816

18. Hoeven L van, Koes BW, Hazes JM, Weel AE (2015) Evaluating the ASAS recommendations for early referral of axial spondyloarthritis in patients with chronic low back pain; is one parameter present sufficient for primary care practice? Ann Rheum Dis 74(12):e68

19. Lambert RG, Bakker PA, van der Heijde $D$, Weber $U$, Rudwaleit M, Hermann KG, Sieper J, Baraliakos X,
Bennett A, Braun J, Burgos-Vargas R, Dougados M Pedersen SJ, Jurik AG, Maksymowych WP, MarzoOrtega $H$, Østergaard $M$, Poddubnyy $D$, Reijnierse $M$, van den Bosch F, van der Horst-Bruinsma I, Landewé $R$ (2016) Defining active sacroiliitis on MRI for classification of axial spondyloarthritis: update by the ASAS MRI working group. Ann Rheum Dis 75(11):1958-1963

20. Linden S van der, Valkenburg HA, Cats A (1984) Evaluation of diagnostic criteria for ankylosing spondylitis. A proposal for modification of the New York criteria. Arthritis Rheum 27(4):361-368

21. Poddubnyy D, Vahldiek J, Spiller I, Buss B, Listing J, Rudwaleit M, Sieper J (2011) Evaluation of 2 screening strategies for early identification of patients with axial spondyloarthritis in primary care. JRheumatol 38(11):2452-2460

22. Poddubnyy $D$, van Tubergen $A$, Landewé $R$, Sieper $J$, van der Heijde D (2015) Development of an ASAS endorsed recommendation for the early referral of patients with a suspicion of axial spondyloarthritis Ann Rheum Dis 74(8):1483-1487

23. Molnar C, Scherer A, Baraliakos $X$, de Hooge $M$, Micheroli R, Exer $P$, Kissling RO, Tamborrini G, Wildi LM, Nissen MJ, Zufferey P, Bernhard J, Weber U, Landewé RBM, van der Heijde D, Ciurea A, Rheumatologists of the Swiss Clinical Quality Management Program (2018) TNF blockers inhibit spinal radiographic progression in ankylosing spondylitis by reducing disease activity: results from the Swiss Clinical Quality Management cohort. Ann Rheum Dis 77(1):63-69

24. Rudwaleit M et al (2009) The development of Assessment of SpondyloArthritis international Society classification criteria for axial spondyloarthritis (part II): validation and final selection. Ann Rheum Dis 68:777-783

25. Rudwaleit $M$ et al (2009) The early disease stage in axial spondylarthritis: results from the German Spondyloarthritis Inception Cohort. Arthritis Rheum 60:717-727

26. Sieper J, Poddubnyy D (2017) Axial spondyloarthritis. Lancet 390(10089):73-84

27. Sieper J, Srinivasan S, Zamani O, Mielants $H$, Choquette D, Pavelka K, Loft AG, Géher P, Danda D, Reitblat T, Cantini F, Ancuta C, Erdes $S$, Raffayová H, Keat A, Gaston JS, Praprotnik S, Vastesaeger N (2013) Comparison of two referral strategies for diagnosis of axial spondyloarthritis: the Recognising and Diagnosing Ankylosing Spondylitis Reliably (RADAR) study. Ann Rheum Dis 72(10):1621-1627

28. Tubergen A van, Heuft-Dorenbosch $L$, Schulpen G, Landewé R, Wijers R, van der Heijde $D$, van Engelshoven J, van der Linden S (2003) Radiographic assessment of sacroiliitis by radiologists and rheumatologists: does training improve quality? Ann Rheum Dis 62(6):519-525

29. Winter J de, de Hooge $M$, van de Sande $M$, de Jong $H$, van Hoeven L, de Koning A, Berg IJ, Ramonda $\mathrm{R}$, Baeten $\mathrm{D}$, van der Heijde $\mathrm{D}$, Weel $\mathrm{A}$, Landewé $R$ (2018) An ASAS-positive MRI of the sacroiliac joints can also occur in healthy individuals, runners and women with postpartum back pain. Arthritis Rheumatol 70(7):1042-1048

30. Zink A, Albrecht K (2016) Wie häufig sind entzündlch rheumatische Erkrankungen in Deutschland? ZRheumatol 75:346-353

31. ZinkA, Braun J, Gromnica-Ihle E, Krause D, Lakomek HJ, Mau W, Müller-Ladner U, Rautenstrauch J, Specker C, Schneider M (2017) Memorandum of the German Society for Rheumatology on the quality of treatment in rheumatology-update 2016. ZRheumatol 76(3):195-207 
Hier steht eine Anzeige.

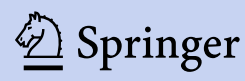

